Historic, Archive Document

Do not assume content reflects current scientific knowledge, policies, or practices. 
.

(1)

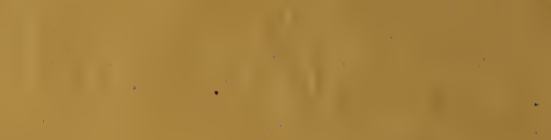

:

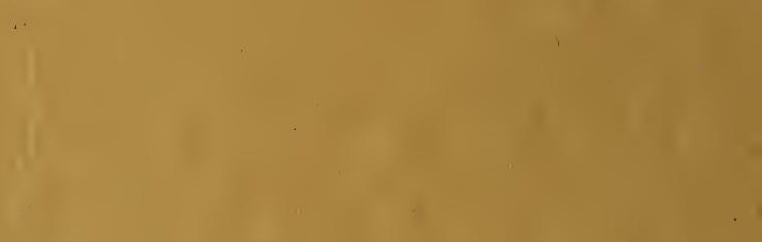
1)

1

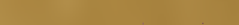

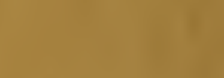

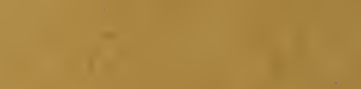

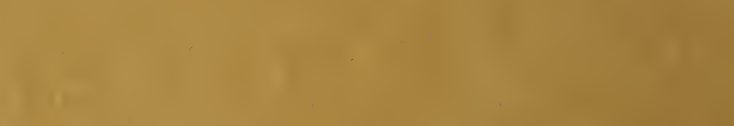

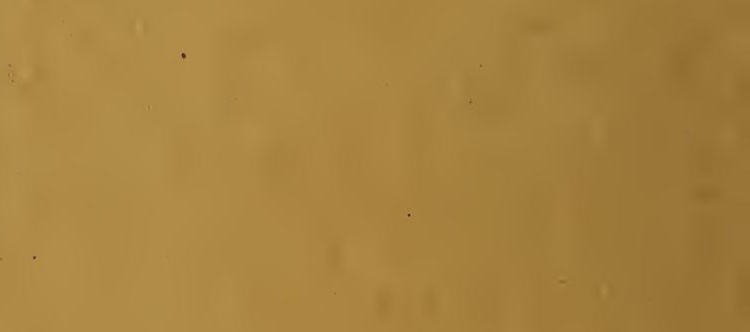

$n+1.6$

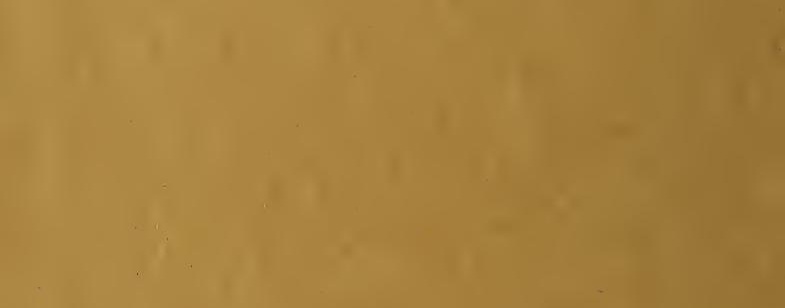

$$
x_{-12}^{2}
$$

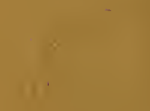




\section{FEB 191917}

RETURN TO POROLOGY

IMDEXEO

ECTION OF NOMELLDAELS:

AWARDED FOR GROWING EXHIBITS OF

SEEDLING DAHLIAS

\section{Silver Medal}

Silver Medal

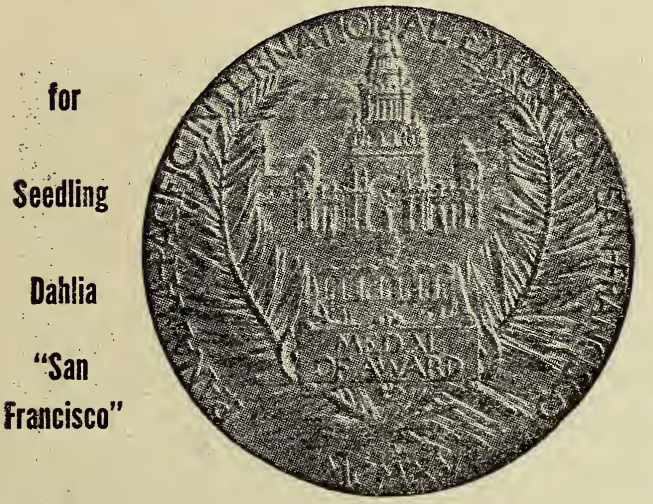

for

GOLD - MED

Seedling

Dahlia

"Mignan"

SEEDLING DAHLIA

"MRS. RICHARD LOHRMANN"

(THE ONLY GOLD-MEDAL AWARDED FOR A SEEDLING)

Silver Medal

Bronze Medal

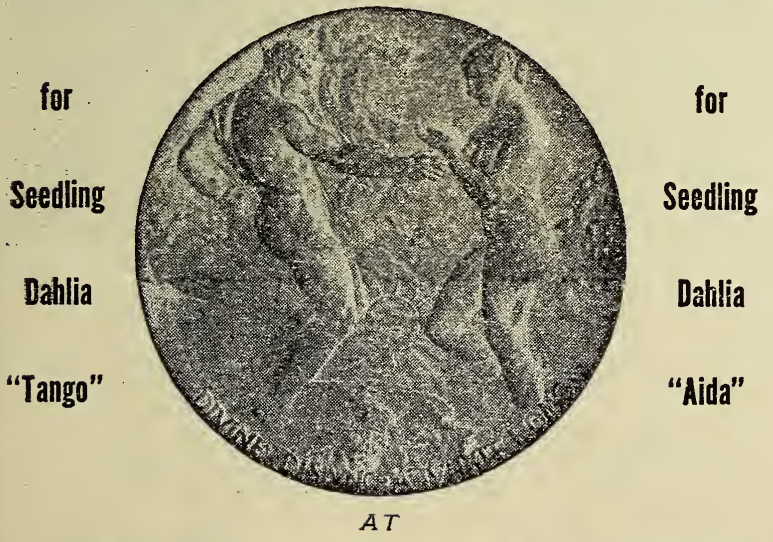

Panama Pacific International Exposition

SAN FRANCISCO, 1915 


$$
\text { GOLD-MEDAL }
$$

AWARDED FOR A GROWING EXHIBIT OF 36 VARIETIES OF $D A H L I A S$

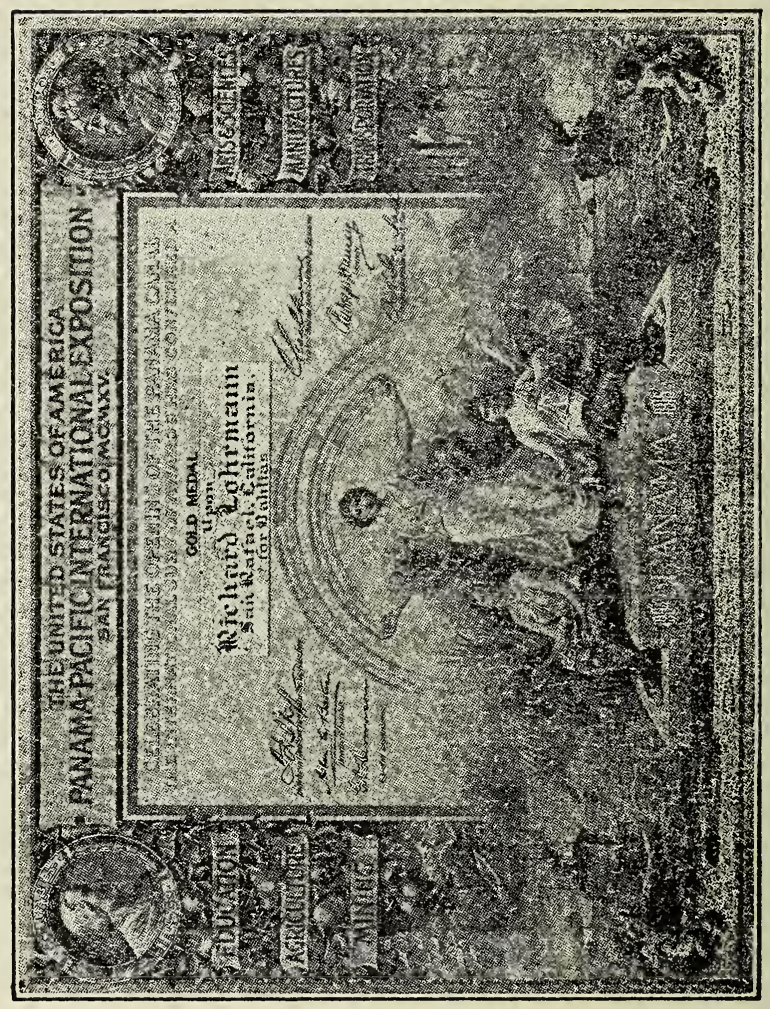

BRONZE MEDAL

AWARDED FOR REHMANNIA ANGULATA

DIPLOMA OF MERIT AWARDED FOR GERMAN IRIS AT

Panama Pacific International Exposition SAN FRANCISCO, 1915 


\section{CHOICE \\ Freit and}

Ornamental Trees

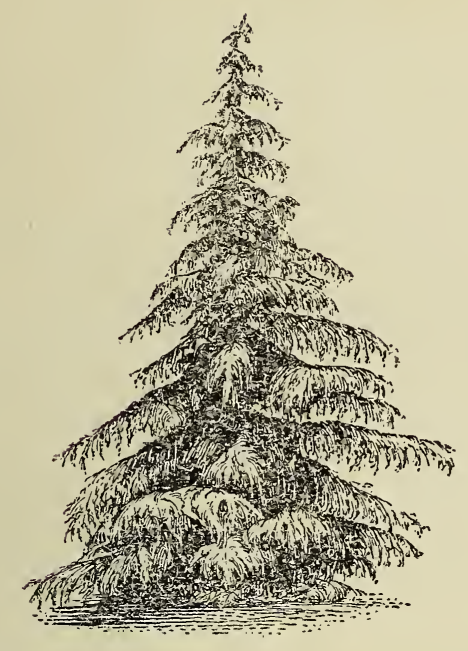

AND

Hardy

Flowering Plants

1917

\section{RICHARD LOHRMANN}

THE WEST END NURSERY

FIFTH AVENUE AND CENTER STREET

SAN RAFAEL - - - CALIFORNIA 


\section{To Correspondents}

I take pleasure in presenting herewith my seventh annual Catalogue and Price-List.

It has been my endeavor, to list, as much as possible, only the very best and most satisfactory of both Fruit and Ornamental Trees and Plants. Whatever I am not able to grow on my own grounds, I procure from the most reliable Nurseries. My Nursery is located between Fifth Avenue and Center Street, about four blocks northwest from West End Station, San Rafael.

I warrant all trees and plants, to be healthy, and true to label, but having no control over them, after delivery, I do not GUARANTEE them to live and thrive, nor will I replace them, except any tree or plant should prove untrue to name; but in no case will I be liable for any sum greater than the original purchase-price.

My customers are requested to notify me immediately of any error, that may have occurred in filling their order, so that the same may be rectified, as I desire to give satisfaction to all, who favor me with their order.

All orders delivered free in San Rafael and Ross Valley; a small charge for packing, to cover cost of material, will be made for all orders shipped by express or freight. All articles are at the risk of the purchaser, after being shipped.

Orders from unknown correspondents must be accompanied by cash or satisfactory reference. All bills payable first of the month.

\section{Landscape Gardening}

Estimates for planting, remodeling old gardens, and laying out new grounds, will be cheerfully given on application.

\section{RICHARD LOHRMANN}

\section{The West End Nursery}

Fifth Avenue and Center St., San Rafael, Cal. Phone S. R. $359 \mathrm{~W}$ 


\section{Fruit Trees}

The varieties listed are the best and most satisfactory for this locality. Prices for new varieties, and larger quantities on application. The trees are 1 and 2 years old, averaging 4 to 6 feet in height.

APPLES:

Each Per 10

Summer: Gravenstein, Red Astrachan, Red June, Bismark.

Fall: Jonathan, R. I. Greening, Alexander, Skinner's Pippin.

Winter: Baldwin, Esopus Spitzenburg, Northern Spy, Yellow Bellflower, Yellow Newton Pippin.

Crab: Red and Yellow Siberian.

PEARS :

Summer: Bartlett.

Fall: Flemish Beauty, Beurre Clairgeau,Doyenne de Comice, Seckel.

Winter: Winter Nelis Winter Seckel, Easter, Pear.

\section{CHERRIES :}

Black Tartarian, Royal Ann, Black Republican, Early Purple Guigne, Bing, English Morello.

\section{PLUMS:}

European var.: Green Gage, Jefferson, Washington, Diamond, Grand Duke, Damson, Yellow Egg.

Jap varieties: Climax, Wickson Satsuma, Santa Rosa.

PRUNES:

Imperial Eplineuse, German, $\$ \quad .35 \$ 3.00$

French, Standlard, Sugar.

\section{PEACHES:}

Freestone: Briggs' Red May, Mayflower, Early Crawford, Opulent, Elberta, Strawberry, Hales Early, Foster, late Crawford, Muir, Salway.

Clingstone: Nichols Orange; and Philipps Cling. 


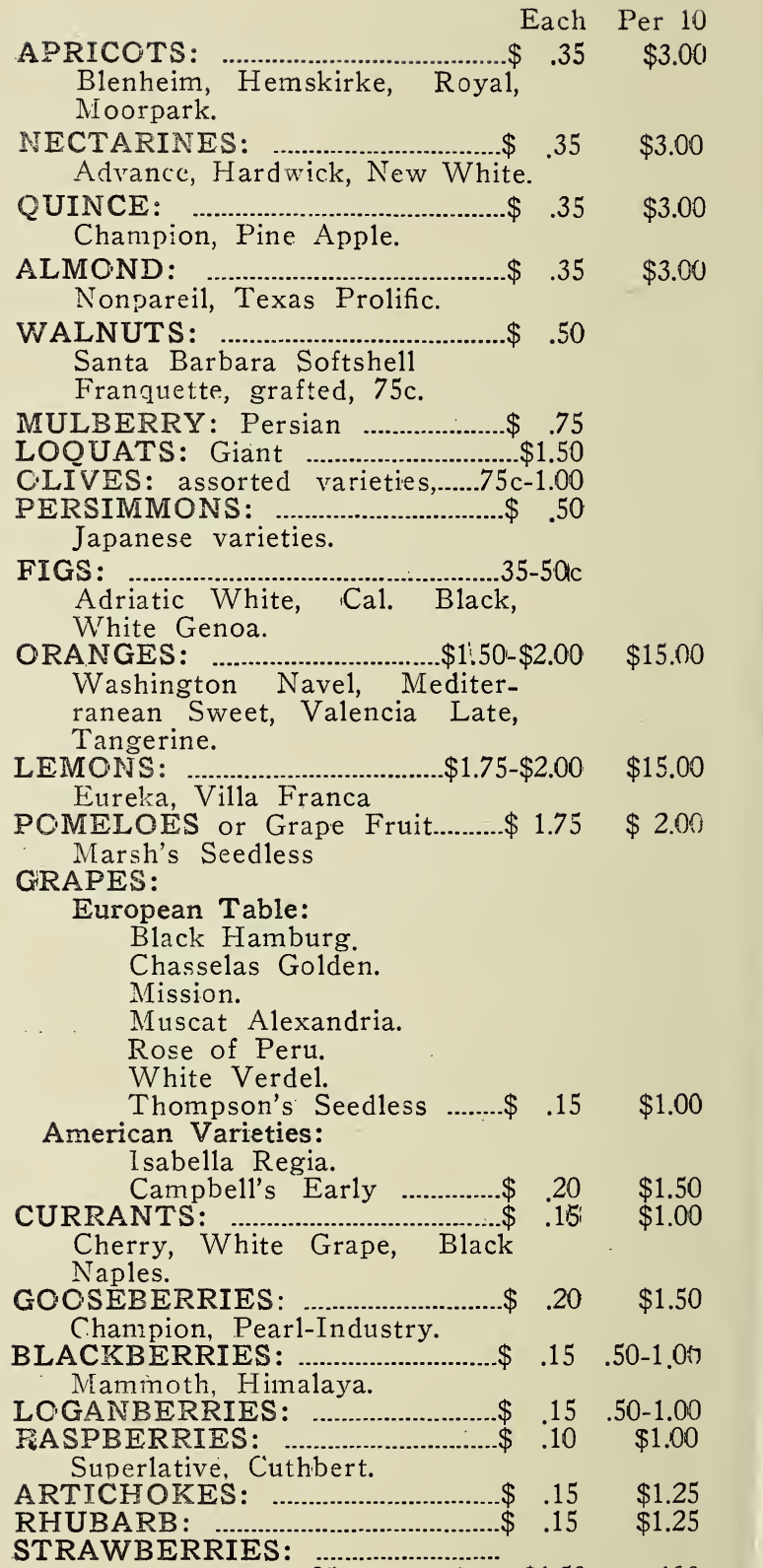




\section{Conifers}

Coniferous Trees are the grandest trees in existence. A good many of them are natives of the Pacific coast, therefore at home in our cli. mate. Though the most expensive varieties are of rather slow growth, until well established in the ground, they will grow into the finest specimen trees for the lawn in a few years. Prices are lfor plants, measuring 1 to 3 feet. Quotations for larger plants on application.

ABIES, Fir: $\quad$ Each

Cephalonica, Silver .....................................\$4.00

Lasiocarpa .....................................................\$3.50

Nordmanniana, Caucasian .........................\$3.00

Pinsapo, Spanish ......................................\$6.00

ABIES (Picea), Spruce:

Pungens glauca, Colo. Blue Spruce $\$ 2.50-5.00$

Exelsa, Norway .....................................50c-1.50

Orientalis $\$ 2.00$

ARAUCARIA:

Bidwilli, Moreton Bay Pine...........\$1.00-\$5.00

Exelsa, Norfolk Island Pine............\$1.00-\$2.00

Imbricata, Monkey Puzzle

$.75 c-\$ 3.50$

\section{CEDRUS:}

Deodara, Himalayan Cedar .............75c-\$3.0C

CRYPTOMERIA:

Japonica elegans ..............................\$1.00-\$3.00

\section{CUPRESSUS:}

Arizonica, Blue Cypress .................\$1.00-\$1.5!)

L.awsoniana, Lawson's Cypress .........50c- $\$ 1.00$

Macrocarpa, Monterey Cypress in flats per 100

Sempervirens, Italian Cypress.... $\quad . . .25 c-\$ 1.50$

Funebris $\$ 1.00-\$ 2.00$

\section{JUNIPERUS:}

Hibernica (Irish) ..................................75c-\$1.25

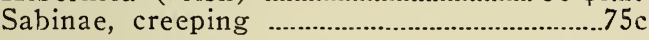

PINUS:

Insignis, Monterey Pine ......................50c-\$2.00

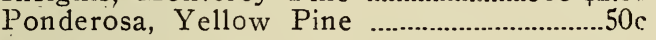

SEQUOYA:

Gigantea, Cal. Big T'rees.................\$2.00-\$4.00

Sempervirens, Redwood

THUYA:

Orientalis, Arborvitae

$.50 c-\$ 1.00$

TAXODIUM:

Distichum, Swamp Cypress .......................\$1.50

TAXUS:

Baccata, English Yew

Baccata fastigiata, Irish 


\section{Evergreen Trees and Shrubs}

Broadleaved Evergreens vary in size from the largest trees to the smallest shrubs; unlike the coniferous Evergreens, whose flowers are insignificant, most of these excel in brilliancy and floriferousness, others through their shining berries and foliage. These plants give our California garden, and Parks their distinctive feature.

ABELIA:

Grandiflora

Each $.50 c-75 c$

ACACIA :

Bayleyana $25 c-50 c$

Cultriformis

Floribunda

Mollissima

Melanoxylon

Verticillata

$.25 c-50 c$

$15 \mathrm{c}-50=$

$.15 d-35 c$

$1,5 c-35 c$ $50 \mathrm{c}$

\section{ARBUTUS:}

Uneedo, strawberry- tree

$75 c-\$ 2.00$

ALECTRYON EXELSUM:

New Zealand Oak

$\$ 1.00$

AUCUBA:

Japonica aurea

$25 c-\$ 2.00$

AZARA:

Microphylla

$35 . c-50 c$

BERBERIS:

Darwini

$25 c-\$ 1.00$

\section{BRACHYCHITON :}

Sterculia acerifol, Flame Tree.................\$1.50

BUDDLEYA:

Veitchiana

$25 c-\$ 1.50$

\section{BUXUS:}

Arborescens, Pyramids

Suffruticosa, Dwf. box, per 100 .

$\$ 1.50-\$ 7.50$

CYTISUS:

Andreara $\$ 3.00$

Alba, Portugal Broom $75 \mathrm{c}$

Scoparius, Scotch Broom ..............................75c

Spartium junceum, Spanish broom......25c-50c

CAMELLIA JAPONICA:

Double White, Pink and Red..........\$1.50-\$3.00

CAMPHOR:

Officinalis, Camphor tree

$50 c$

\section{CASUARINA:}

Stricta

$\$ 1.00-\$ 2.00$

CHOYSIA TERNATA:

CARPENTERIA:

$25 c-\$ 1.00$

Californica 
CISTUS:

Assorted Varieties ...............................25c-50c

CORNUS CAPITATA:

Evergreen Dogwood ..................................\$1.00

COTONEASTER:

Francheti .......................................................... \$1.75

Angustifol: orange Berries .........................50c

Pannosa, vermilion red berries........25c- $\$ 2.00$

Nepalensis, showy, red ..........................\$1.50

Microphylla, creeping .............................50c- $\$ 1.00$

Horizontalis, bright red Berries.................\$1.00

CRATAEGUS:

Pyracantha, Orange Berries................50c- $\$ 1.50$

Crenulata, Scarlet Berries ...................75c-\$1.25

DAPHNE:

Odora, pink and white......................\$1.00-\$2.00

DIOSMA :

Alba, Breath of Heaven.........................35c-75c

ELAEGNUS:

Reflexa aurea .........................................35c-50c

ESCALLONIA:

Rosea

$.50 c-\$ 1.00$

Montevidensis, white, $.50 \mathrm{c}-\$ 1.00$

EUCALYPTUS:

Globulus Blue Gum in pots.................10c-15c

Globulus Blue Gum in flats, per $100 \ldots \ldots . . . \$ 2.00$

Ficifolia, scarlet-flowering ...........................5c-50c

Salicifolia, willow-leaved ...........................25c-50r

Assorted Varieties ...................................25c-50c

EUGENIA:

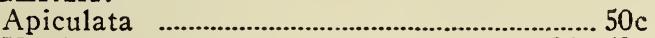

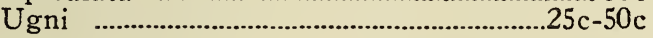

FICUS:

Macrophylla, Moreton Bay Fig.......\$1.00-\$1.50

GREVILLEA :

Robusta, Silk Oak ..............................50c-75c

ERICA :

Mediterranean Heath, Purple ............25c-\$1.00

Persoluta alba, white ............................50c-75c

Melanthera, pink .................................................

Cinerea Carnea

$35 c-50 c$

ILEX Aquifolium

English Holly, berried plants........\$1.00-\$7.50

EVONYMUS:

Japonica aurea

$25 c-\$ 1.00$ 
HYPERICUM:

Moserianum, golden yellow

$.50 \mathrm{c}$

KALMIA:

Latifol: Mountain Laurel

$\$ 2.00$

LAURUS:

Laurocerasus, English Laurel

$50 c-\$ 2.00$

Lusitanica, Portugal Laurel

$\$ 1.50-\$ 2.00$

LAURUSTINUS $75 \mathrm{c}$

\section{LIGUST RUM:}

Ovalifolium, Cal. Privet, per 100....\$5.00-\$7.00

Sinensis, Chinese Privet

LYONOTHAMNUS:

Floribundus, Ironwood

\section{LEPTOSPERMUM:}

Laevigatum

Nicholssi, red flowering

$.75 \mathrm{c}$ $.75 c-\$ 1.50$

MAHONIA:

Aquifol: Oregon Grape $\$ 1.00$

MAGNOLIA:

Grandiflora $.50 c-\$ 1.50$

MELALEUCA :

Hypericifolia $.50 c$

MYRTUS:

Communis, English Myrtle ...........................50c

Communis, microphylla, Bridal Myrtle....5.0c

NANDINA:

Domestica, crimson berries …………........75c

OSMANTHUS: Aquifol: ........................................

OLEANDER:

Assorted Varieties ............................. $\$ 1.00-\$ 2.00$

PITTOSPORUM:

Eugenoides …............................................25c-\$1.00

Tobirae ….................................................... $50 \mathrm{c}$

Undulatum ……..................................... \$1.00

POLYGALA:

Dalmaisiana, continuous bloomer.........25c-35c RHODODENDRON:

Hybrids, the finest varieties............ $\$ 2.00-\$ 3.00$

Pink Pearl

$\$ 3.00-\$ 5.00$

SCHINUS MOLLIS:

Cal. Pepper

$25 c-50 c$

VERONICA:

Imperialis

Decussata 


\section{Deciduous Trees and Shrubs}

The best street and avenue trees are decidely deciduous trees; they will give the necessary shade in summer and admit sunshine in winter. In this class are also the most gorgeous spring and summer blooming shrubs. Deciduous weeping trees are beautiful specimen trees for the lawn.

APPLE:

Each

Ionensis, Bechtels, double Rose....................50c

Spectatilis fl. pl. ................................................5fic

ALMOND:

Double Pink dwarf ..........................................50c

BEECH:

Copper or purple leaved............................... $\$ 1.0^{\prime}$

BERBERIS:

Thunbergii .......................................................... 35

Common and Purple-leaved........................

\section{BIRCH:}

Cutleaved Weeping …...........................50c- $\$ 1.00$

European white

CATALPA:

Bignonioides ……….................................. $75 c-\$ 1.00$

\section{CHERRY:}

Jap., weeping ..................................................\$2.00.

CEANOTHUS:

Gloire de Versailles ........................................50

\section{CERCIS:}

Canadensis, Judas Tree

$.50 \mathrm{c}$

\section{CYTISUS LABURNUM:}

Golden Chain $75 c$

DEUTZIA:

Assorted Varieties

$25 c-50 c$

\section{ELM:}

American, a magnificent tree ..................... $\$ 1.00$ EXOCHORDA:

Grandiflora, Pearl Bush.................................50

FILBERT, purple leaved $.50 c-\$ 1.00$

FORSYTHIA:

Intermedia, Golden Bells ................................35c

GINGKO BILOBA:

Maiden-hair Tree

$75 \mathrm{c}$

HORSE-CHESTNUTS:

Red flowering

$\$ 1.00-\$ 1.50$ 
HYDRANGEA :

Hartensis

Each

Hortensis

$10 \mathrm{c}-25 \mathrm{c}$

New French Varieties, assorted........25c- $\$ 2.00$ KERRIA :

Japonica, single and double

$25 \mathrm{c}-50 \mathrm{c}$

I.EMON VERBENA:

$2.5 \mathrm{c}-50 \mathrm{c}$

\section{LILAC:}

Syringa vulgaris, purple and white.............50c Syringa, assorted grafted varieties......50c-75c

\section{LINDEN :}

Eulropean $\$ 1.00$

LAGERSTROEMIA Indica:

Pink Crepe Myrtle

LOCUST :

Pink and Rose flowering

$.75 c-\$ 1.00$

MAPLE:

Acer Schwedleri, Purple-leaved.................\$1.00

Acer dasycarpum, Silver

$.75 \mathrm{c}$

MOCK-ORANGE:

Philadelphus grandiflorus $35 \mathrm{c}$

MOUNTAIN ASH: $\$ 1.00$

PEACH :

Double Red,. White, Pink.

$50 c-75 c$

\section{PLUM:}

Purple leaved

$50 c-75 c$

Vesuvius, (Burbank's) $75 \mathrm{c}$

Moserii fl-pl., double

$75 \mathrm{c}$

POPLAR:

Lombardy and Carolina $.50 \mathrm{c}$

POINCIANA GILLESI:

Bird of Paradise shrub.

$35 c-50 c$

POMMEGRANATE:

Double Scarlet

$35 c-50 c$

PYRUS ARBUTIFOLIA:

Bright red berries

$.50 \mathrm{c}$

QUINCE:

Japanese, scarlet $.50 \mathrm{c}$

SNOWBALL:

Common

$35 c-50 c$

SPIRAEA:

Bridal Wreath, assorted varieties..................50c

Anth, Waterer, Pink $35 c$

SYCAMORE:

Oriental, the finest Avenue tree.

$\$ 1.00$

THORN :

Double, Red, White, Pink......................50c-\$1.00

Cordata, Washington .................................. $\$ 1.00$

TAMARIX. Africana

$35 \mathrm{c}-50 \mathrm{c}$

WEIGELIA :

Assorted Varieties

WILLOW Babylonian

$35 c$

$.50 c-\$ 1.00$ 


\section{Climbing Plants}

A most useful class of plants, for covering bare walls, trellises, unsightly fences, to plant on verandas and pergolas, in rockeries and as a ground cover under trees.

\section{AMPELOPSIS:}

Quinquefol: Virginia Creeper ....................2.5c

Veitchii: Boston Ivy .....................................25c

Stricta sempervirens .......................................50

BIGNONIA:

Radicans grandiflora, Trumpet Vine........25c

Cherere, blood red .............................................25 c

BOUGAINVILLEA:

Spectabilis, magenta flowers ............50c- $\$ 1.00$

\section{CLEMATIS:}

Montana, white ..........................................35c.

Crispa, small lavender flowers.......................35c

Jackmanni, large flowering varieties............75c

FICUS: repens

\section{HONEYSUCKLE:}

Lonicera Halleana, white and yellow..15c-25c

Lonicera Heckrothii, rosy purple..............25c

IVY:

English. Rotted cuttings per $100 \ldots \ldots . . . \$ 1.50$

JASMINUM:

Officinale, white fragrant

Revolutum, yellow

$35 c$

Grandiflorum, double white

Primulinum, double yellow

$15 c-75 c$

$.50 \mathrm{c}$

$15 c-35 c$

MANDEVILLEA:

Suaveolens, white

$.35 c$

MUHLENBECKIA COMPLEXA:

Maidenhair Vine $10 c$

PASSION VINES:

Passiflora, cocrulea, blue ............................25c

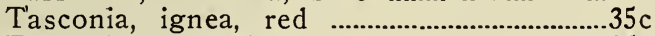

Tasconia mollissima, pink .............................35c

PEA VINE:

Australian

$25 c$

PLUMBAGO:

Capensis, azure blue

$15 c$

SOLLYA HETEROPHYLLA:

Austral: Blue Bells

TECOMA:

Jasminoides, rosy pink

Smithii, orange-yellow

$.50 c-75 c$

$25 \mathrm{c}$

VITIS: capense $25 \mathrm{c}$

\section{WISTARIA:}

Chinensis, purple and white

Multijuga, dark purple and white........75c-\$1.00 


\section{Palms, Ferns and Foli- age Plants}

Hardy Palms are undoubtedly the most decorative plants in our garden; a group of several varieties, including plants of similar habit will give the landscape a tropical appearance. Prices are for smaller plants, potted or balled; quotations for large specimens and varieties not mentioned on application.

CHAMAEROPS:

Humilis, Dwarf Fan Palm $\$ 2.00$

Exelsa, Japanese Fan Palm $\$ 2.00-\$ 3.50$

COCOS AUSTRALIS:

$A$ very graceful hardy $\mathrm{Palm}$ $\$ 3.00-\$ 5.00$ ERYTHEA EDULIS:

Guadeloupe [sland Palm

Armata, Blue Palm

$\$ 2.00-\$ 5.00$

$\$ 4.00-\$ 6.00$

PHOENIX CANARIENSIS:

Canary Island Date Palm........................50c- $\$ 4.00$

KENTIA BELMOREANA:

The best of the House Palms

$.50 c-\$ 5.00$

WASHINGTONIA:

Robusta, California Fan Palm....................\$1.50

Sonorae. one of the best Fan Palms........\$2.00

ADIANTUM cuneatum:

Maiden-hair Fern

$25 c-75 c$

ADIANTUM PEDATUM:

Five Finger Fern, hardy

$.25 \mathrm{c}$

ASPLENIUM nidus avis:

Bird's-nest Fern

BOSTON FERNS:

Assorted Varieties

CYRTOMIUM FALCATUM:

Jap. Holly-Fern

PTERIS:

Small Ferns for Fern dishes.......................15c WOODWARDIA FERNS ASPIDISTRA, LURIDA: BANANA, Abyssinian

BAMBOO, Clumps CALADIUM ESCULENTUM:

Elefants-Ears

$\$ 1.00-\$ 2.00$

$.25 \mathrm{c}-\$ 3.50$

DRACAENA:

Australis and Indivisa

EULALIA Zebrina stricta:

7.ehra Grass

ASPARAGUS:

Plumosus

$5 n c$

$50 c-\$ 1.00$

$50 c-\$ 1.00$

$50 \mathrm{c}-\$ 1.25$

Sprengerii, in pots

Sprengerii, in baskets

LOTUS BERTHOLEBI:

$25 \mathrm{c}$

Fine for Window boxes and hanging baskets 


\section{Roses}

Price for field-grown plants, 25 cents each; $\$ 2.00$ per $10 ; \$ 18.00$ per 100 ; except for Novelties and newer introductions.

\section{I}

TEA AND HYBRID PERPETUAL ROSES

American Beauty, deep rose.

Bride: white.

Bridesmaid: pink.

Betty, coppery rose.

Duchesse de Brabant, silvery, pink.

General McArthur, fiery red.

Frau Karl Druschki, snow-white.

General Iacqueminot; red.

Hugh Dickson, brilliant crimson.

Jonkher, L. Mock, salmon pink.

J. B. Clark; intense scarlet.

Kaiserin Augusta Victoria; cream-white.

Laoy Alice Stanley, coral rose.

La France, silvery rose.

Lady Hillingdom, orange-yellow.

Mme. Caroline Testoute, pink.

Mlle. Cecile Brunner, salmon-pink.

Melody, canary-yellow

Maman Cochet, pink.

Maman Cochet, white.

Milady. crimson scarlet ..............................35c

Niles Cochet, red.

Mrs. Geo. Shawyer, klear rose.

Mrs. John Laing, pink.

Papa Gontier, rosy-crimson.

Paul Neyron, dark rose.

Perle des Jardin, golden yellow.

Prince C. de Rohan, dark crimson.

Rhea Reid, velvety red.

Richmond, dark crimson.

Sunburst, orange coppery yellow.

$35 c$

Ulrich Brunner, cherry-red.

Mrs. Wakefield Christie Miller, blush-pink..

\section{NOVELTIES}

ANTOINE RIVOIRE,

Silvery flesh, salmon center..........................50c BEAUTY DE LYON:

Coral red, shaded yellow

FARBEN-KOENIGIN :

Bright silvery carmine .............................\$1.00

JULIET :

Old gold. rosv red to deep rose................75c GEORGE DICKSON:

A dark velvety crimson, of extra ordinary size and rare perifume........each $50 \mathrm{c}-75 \mathrm{c}$ GEO. AHRENDS:

Bright red carmine 
OPHELIA:

Salmon flesh shaded rose.............................\$1.00

MME. ED. HERRIOTT:

Coral red, shaded yellow, changing to shrimp pink ....................................................75c

PRIMADONNA:

Deep Rose-pink .........................................50 c

RAYON d'OR:

Golden yellow, tinged with coppery or-

ange

\section{II}

\section{CLIMBING ROSES}

Climbing American Beauty, NEW. $35 c$

Climbing Belle Sicbrecht, bright rose.

Climbing Bridesmaid, pink.

Climbing, Cec. Brunner, salmon pink.

Climbing Perle des Jardin, golden yellow.

Climbing Kaiserin Aug. Victoria creamy white.

Climbing Papa Gontier, rosy crimson.

Climbing Caroline Testoute, pink.

Climbing Souvenir de Wootton, bright crimson.

Climbing Pink M. COCHET; NEW........35'c

La Marque, white.

Marechal, Niel, yellow.

Reve d'Or, coppery-yellow.

The following varieties flower only in the Spring:

American Pillar, single pink, white eye.

Banksia, yellow.

Banksia, white.

Cherokee, double white.

Cherokee, single white.

Cherokee, pink, single.

Dorothy Perkins, shell-pink.

Lady Gay; cerise pink.

Hiawatha, crimson-white center.

San Rafael, or Beauty of Glazenwood.

RAMONA, single carmine-crimson.

Tausendschoen, delicate pink, NEW;.....35c

\section{III}

MISCELLANEOUS ROSES.

Jessie, cherry crimson, dwarf.

Hybrid Sweet Briars, single rose, crimson and white.

Moss Roses, pink, red and white.

Austrian Copper, single.

Persian Yellow, double yellow.

pink

Irish Elegance, apricot, shaded orange and

IRISH FIREFLAME:

Old Gold, flushed with pure crimson, large single flowers, NEW

\section{IV}

STANDARD ROSES

Assorted Varieties, \$1.25 each; $\$ 10.00$ per 10. 


\section{Dahlias}

The following list of Dahlias comprises the cream of numerous varieties in commerce. They have been selected for their superiority in purity of color, perfection of form, length of stem, continuous blooming, etc.

Abbreviations: C-Cactus; D-Decorative; Sh-Show; Pae-Paeonia flowering; F-Fancy.

\section{California Dahlias of my own intro- duction}

I beg to draw my customers special attention to my own introductions, which were planted and exhibited at the Panama-Pacific International Exposition in San Francisco.

AIDA-D. (Bronze Medal.)

Dark maroon, large, ......................................50c

ALOHA-C.

Light pinkish coral

$.35 \mathrm{c}$

ELSA-Pae.

Fluffy white, Flowers, yellow, center,

large size,

FATINITZA-F. C.

Light purplish pink, mottled crimson.........35

GIRL of the GOLDEN WEST-Pae.

Light yellow, tinged and tipped with red $75 \mathrm{c}$ ISOLDE-C. D;

Creamy white, recurved petals......................50 c

MIGNON-C. Pae. (Silver Medal)

Delicate lavender-pink $.50 \mathrm{c}$

MRS. RICHARD LOHRMANN-D.

A pure golden yellow, of immense size, full center, broad curly petals; awarded Gold Medal at the Panama-Pacific International Exposition $\$ 1.00$ each

ORTRUD-D. Sh. Extra large flower, delicate pale lavender $50 \mathrm{c}$

SAN FRANCISCO-C. D. (Silver Medal) Immense flowers of a reddish bronze carried high above the foliage on upright stems

SALCME JANE-C. D.

Old gold, tinged with red SENTA-C. D.

Large Dure white flowers $.50 \mathrm{c}$ TELRAMUND-Pae.

Carmine-red with pink tips

TANGO-Pae. (Silver Miedal)

Large salmon colored flowers, prolific

bloomer, and a promising cut-flower

variety 


\section{General Collection}

(Complete List on Application)

\section{BELOIT:-D}

Purplish-garnet

BLACK DIAMOND:-Sh

Deep velvety maroon

DELICE:-D

Rose-pink

$25 c$

GOLD-MEDAL:-D. Sh.

Golden-yellow, dotted red ...........................25c

GEISHA:-Pae.

Scarlet and gold...................................................35c

HORTULANUS FIETH:-D

Immense blooms of a shrimppink..................50 c

KAISER WILHELM:-Sh.

Large yellow ,tipped with red.....................25c

KALIF:-C.

Pure scarlet, a giganticflower

$50 \mathrm{c}$

MARGUERITE BOUCHON:-C.

Soft rose, white in the center and white tips

$50 \mathrm{c}$

MINNIE BURGLE:-D.

A rich glowing crimson.

$25 c$

NIBELUNGEN-HORT :-C.

Old rose, suffused with golden apricot....50c NUGGETT:-Sh.

Old gold, overlaid with red $.25 \mathrm{c}$

PERLE DES LYON:-C. D.

White

$25 \mathrm{c}$

RHEINGAU:-C. Pae.

Brilliant scarlet $.25 \mathrm{c}$

RHEIN-TOCHTER:-C.

Mauve-pink

$25 \mathrm{c}$

SOUVENIR DU G. DOAZON:-D.

Pure scarlet, a mammoth flower $.25 c$

VATER RHEIN-C. Pae.

Yellow, suffused with salmon-rose..............35c

WACHTAM RHEIN:-C.

Rosy-pink

WALKUERE:-C.

Very large sulphur-yellow

$35 c$

WODAN:-C.

Salmon-rose, shading to old gold. $.25 \mathrm{c}$

YELLOW COLOSSE:-D. Sh.

Immense pure yellow 


\section{Hardy Perennials}

Herbaceous plants are indispensable for filling out and planting amongst shrubbery, in the rock garden, for the old-fashioned perennial border, etc. Most varieties, after once planted, will live and bloom without much attention, for many years. Prices are for field grown plants.

per per

doz $\quad 100$

ASTER, perennial

$\$ \quad .60 \$ 4.00$

ANCHUSA,

Italica, Dropmore variety

AQUILEGIA, Columbine:

Chrysantha, yellow .................... $\quad .7 .5 \quad 5.00$

Coerubea, blue ................................. $1.00 \quad 7.00$

Hybrids, mixed colors .................. $\quad .75 \quad 5.00$

BELLIS PERENNIS:

Daisy, red and white.................... $.25 \quad 1.50$

CAMPANULA:

Persicifolia, Bellflower ............. $75 \quad 5.00$

Medium, Canterbury Bells, blue, $75 \quad 5.00$

COREOPSIS:

Grandiflora, golden yellow ....... $.60 \quad 4.00$

DORONICUM caucasicum .............. 1.00

DELPHINIUM Larkspur

Hybrids, mixed varieties............ $1.00 \quad 7.00$

DIANTHUS BARBATUS:

Sweet William ............................. $\quad .50 \quad 3.00$

DIANTHUS PLUMARIUS:

Border Pinks .............................. .60 $\quad .60$

DIGITALIS, FOXGLOVE:

Maculata superba ........................ $\quad .60 \quad 4.00$

GAILLARDIA:

Grandiflora. ..................................... .75 $\quad .00$

GEUM atro purpureum:

Mrs. Bradshaw, double scarlet $1.00 \quad 7.00$

\section{GERBERA JAMESONI HYBRIDA}

(The Giant Transvaal Daisy)

The blossoms of this beautiful Marguerite are 3 to 4 inches in diameter, of an intense vermillion color in the original type; and a series of colors, including pure yellow, orange salmon, rose and ruby red, amongst the Hybrids; unsurpassed as a cut flower. Price 25-50 cents each. HCLLYHOCKS:

Double, mixed varieties

Double, separate colors 
IRIS GERMANICA:

Purple and white ....................... $.60 \quad 4.00$

Isolene, pale lavender, overlaid with brown, NEW; 25 cents each.

Pallida dalmatica, lavender....... 2.50

New Varieties, Fro, Nothung, Mithras, Princess: Vict. Luise, Rheim-Nixe,

Diploma of Merit at the P. P.

I. Exposition

Nibelungen

Assorted older varieties. $\$ 1.00$ per doz.

IRIS INTERREGNA:

Gerda, Halfdan, Walhalla, Ingeborg, a new type of Iris, flowering very early $2.00 \quad 14.00$

IRIS Japanese:

Assorted colors 2.00 14.00

LAVENDER 1.00

\section{LEONOTIS LEONURUS:}

Liontails 100

MYOSOTIS, Forget me not. 1.50

PAECNIA 25.

Finest assorted var. 50ic each, $\$ 5.00$ per doz. PAPAVER:

Oriental, rosy-salmon 1.00

7.00 PENTSTEMON :

Grandiflora, Hybrids .................... $\quad .60 \quad 4.00$

PRIMULA:

Veris, Prim Rose

Malacoides, lavender

PYRETHRUM:

Rosum grandifl.

$\begin{array}{ll}.50 & 3.00 \\ .50 & 3.00\end{array}$

REHMANNIA ANGULATA:

Large dark rose colored flowers $1.00 \quad 7.00$

Well adopted for this climate, blooming all summer; a warded a bronze medal at the P. P.

I. Exposition.

RUDBECKIA:

Single

Golden Glow .75

SCABIOSA causasica .50

SHASTA DAISY:

Alaska 1.00

\section{VIOLA CORNUTA:}

Light-blue

VIOLET:

Princess of Wales 


\section{Summer Flowering Bedding Plants}

These plants are mostly Annuals, raised every year from seed or cutting, several are of a hardier nature, and under favorable conditions will stand our California winters. Prices are for plants, grown in boxes or 2 -inch pots. Ready to plant after April 1st.

ASTER:

$\begin{array}{ll}\text { per } & \text { per } \\ \text { doz } & 100\end{array}$

Branching, separate colors ........ \$ .25 $\$ 1.50$

ANTIRRHINUM:

Snapdragon, mixed ..................... .25 $\quad .25$

BEGONIA:

Semperflorens .50

Tuberous, single ..................................... 1.00

Tuberous, double ......................... 1.50

Tuberous, Crested Varieties...... 2.00

Potted plants each 25c-50c.

\section{CALLIOPSIS:}

Golden Wave

CENTAUREA, CORNFLOWER:

Emperor William

\section{CELOSIA:}

Coxcomb and Ostrich Plume.... $\quad .50 \quad 3.00$

CALCEOLARIA:

Large flowering Hybrids............ 1.00

\section{CHRYSANTHEMUM:}

Named Choice Varieties............. $1.00 \quad 7.00$

Mixed Varieties ............................... $\quad .50 \quad 3.00$

Single and Pompon varieties.... $\quad .50 \quad 3.50$

CINERARIA hybrida grandfl, pot

grown plants

cosmos:

Lady Lenox, pink and white.... .25

\section{GLADIOLUS:}

Named choice varities ................. 1.00

Mixed

GAZANIA SPLENDENS:

\section{GERANIUM:}

Double and single, crimson...... $\quad .50 \quad 3.00$

Ivy leaved, pink and red.............. $\quad .50 \quad 3.00$

\section{HELIANTHUS:}

Sunflower, double Mammoth.... $\quad .50$ 
LOBELIA:

Chrystal Palace, dark blue......... $.25 \quad 1.50$

Sapphire, deep blue, white eyes $\quad .35 \quad 2.00$

MARGUERITES:

White and yellow ....................... $.50 \quad 3.00$

Mrs. F. Sander, double white.. $.50 \quad 3.00$

MARIGOLD:

African, yellow ............................ .25 $\quad .250$

French, mixed colors, dwarf.... $\quad .25 \quad 1.50$

NIGELLA, Love in a Mist

Miss Yekyll ................................. .35 $\quad .00$

PANSIES:

Extra fine mixtures …….............. $.35 \quad 2.00$

PELARGONIUM:

Lady Washington ge ranium,

new, everblooming varieties

each $25 \mathrm{c}$

\section{PETUNIAS:}

Double mixed, extra ….............. $\quad .50 \quad 3.00$

S"rigle, choice varieties .................. . . 35 2. 10

PRIMULA OBCONICA:

Small Plants .................................. 1.00

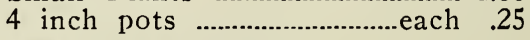

SALVIA SPLENDENS:

Bonfire ............................................ ..50

Patens, blue ………….................... 1.00

STOCKS:

White, red, pink, and mixed...... $\quad .25 \quad 1.50$

SALPIGLOSSIS:

Superbissima: Emperor ….......... $\quad .35 \quad 2.00$

SCHIZANTHUS:

Wisetoniensis, PoorMan'sOrchid .35 2.00

VERBENA:

Assorted colors ............................. .35 2.00

WALLFLOWER:

Double .......................................... .25 1.50

\section{ZINNIA:}

Grandiflora-Robusta, Mammoth $.35 \quad 2.07$

DAHLIA IMPERIALIS:

The Mexican Tree-Dahlia grows to a height from 8 to 10 feet; foliage very ornamental; the light pink bell-shaped flowers appear in November; for this reason the plant is highly recommended for the warmer parts of the United States. Price per bulb. 


